\title{
Polarization-Based Illumination Detection for Coherent Augmented Reality Scene Rendering in Dynamic Environments
}

\author{
A'aeshah Alhakamy ${ }^{1,2}[0000-0002-0662-0185]$ \\ and Mihran Tuceryan ${ }^{10000-0003-3828-6123]}$ \\ 1 Indiana University - Purdue University Indianapolis (IUPUI), USA \\ aalhakam@iupui.edu/@iu.edu/@purdue.edu ; tuceryan@iu.edu \\ https://www.cs.iupui.edu/ tuceryan/ \\ 2 University of Tabuk in Tabuk, Saudi Arabia \\ https://www.ut.edu.sa/en/web/u12562
}

\begin{abstract}
A virtual object that is integrated into the real world in a perceptually coherent manner using the physical illumination information in the current environment is still under development. Several researchers investigated the problem producing a high-quality result; however, pre-computation and offline availability of resources were the essential assumption upon which the system relied. In this paper, we propose a novel and robust approach to identifying the incident light in the scene using the polarization properties of the light wave and using this information to produce a visually coherent augmented reality within a dynamic environment. This approach is part of a complete system which has three simultaneous components that run in real-time: (i) the detection of the incident light angle, (ii) the estimation of the reflected light, and (iii) the creation of the shading properties which are required to provide any virtual object with the detected lighting, reflected shadows, and adequate materials. Finally, the system performance is analyzed where our approach has reduced the overall computational cost.
\end{abstract}

Keywords: Augmented and Mixed Environments · Interaction Design - Scene Perception · Texture Perception

\section{Introduction}

A realistic and immersive experience in augmented reality is one of the problems in computer graphics that still has major limitations and challenges. The main approach is to extract the illumination data in the physical environment and use it to render the virtual objects inserted into the final scene. An illumination model that renders the scene to be perceived as realistic can be challenging if some assumptions are not made. Several innovative techniques are required to render a realistically dynamic scene in augmented reality. In this paper, we propose a new system that (1) uses the properties of polarized light to detect the incident lighting (direct illumination) in the scene, (2) estimates the reflected

This is the author's manuscript of the article published in final edited form as:

Alhakamy, A., \& Tuceryan, M. (2019). Polarization-Based Illumination Detection for Coherent Augmented Reality Scene Rendering in Dynamic Environments. In M. Gavrilova, J. Chang, N. M. Thalmann, E. Hitzer, \& H. Ishikawa (Eds.), Advances in Computer Graphics (pp. 3-14). Springer International Publishing. https://doi.org/10.1007/978-3-030-22514-8_1 
lighting (indirect illumination), and (3) creates the shading properties to apply the previous characteristics on each virtual object material. The system composites seamlessly the three major components of the $3 \mathrm{D}$ virtual object into the live main camera of the AR device. The live-feed of the panoramic $360^{\circ}$ camera captures omnidirectional views as an input from the surrounding mediums. Any AR device (e.g., head mounted display, projection display, handheld mobile, or webcam camera) can be instrumented with such a camera.

The first component to tackle in this problem is the detection of incident light where the light falls directly from the light source onto the objects. An improvement to the method of detecting the direct illumination source was made through the use of polarization filters. Such filtering reduced the unwanted reflections and glares on the radiance maps [8]. The live-feed from the $360^{\circ}$ camera was used for detection after being subjected to polarized filtering. The second component focuses on the estimation of the reflected light where the reflected lights bounce off the surfaces between the objects whether real or virtual (see Figure 1). A 2D texture of the

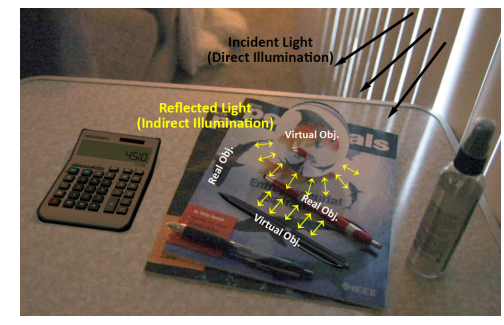

Fig. 1. An example showing the difference between the direct incident light (direct illumination) and the indirect reflected light (indirect illumination). local area surrounding each $3 \mathrm{D}$ object is extracted from the main view of the AR to be uploaded in the Image-Based Lighting (IBL) property of each object as needed. The third component applies the detected direct illumination angle along with the corresponding shadow, and the indirect illumination while creating the shading properties required for each object based on the material features. The lighting conditions, camera position, and object location can be changed in real-time effortlessly. Finally, the differential rendering [5] is used to composite the computer-generated objects with the physical lighting in AR. The resources and algorithms for IBL have been optimized to provide a $360^{\circ}$ live-feed as a direct light source.

\section{Related Work}

\subsection{Use of Polarized Light in Computer Vision}

The art of photography influenced the idea of polarization to be used in this research (see Figure 2). Physics-based computer vision was advanced when Horn introduced the optical models of reflection and imaging in (1975) [1]. Polarized light is more than a human perception of intensity and color; the information about polarization-based vision go beyond vision based on intensity or shading. Chen and Wolff [6] presented a theoretical development and application for material classification using polarization phase-based method according to intrinsic 
electrical conductivity. Also, Ngo Thanh et al. [15] presented a shape reconstruction technique which used shading and polarization with one constraint for both: a pair of light directions for shading, and a pair of polarizer angles for polarization. Shen et al. [19] developed a scheme for dehazing and denoising unclear images where polarizing images on different days were collected to verify the noise reduction algorithm and details optimization.
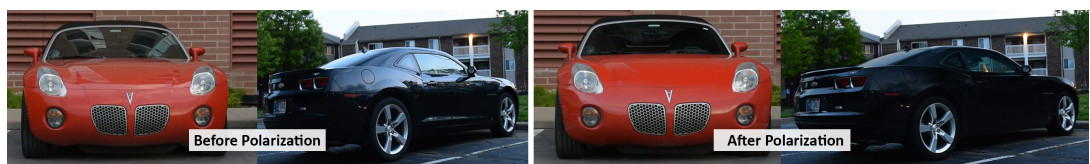

Fig. 2. Illustration how the Circular Polarizer/Linear (CPL) camera filter used to reduce reflections and glare.

\subsection{Incident Light Sampling and Detection}

The physical environment map is captured in order to illuminate the virtual object, known as Image-Based Lighting (IBL) [5]. Due to the low performance of sampling the environment map for a realistic IBL with correct shadows, the procedure is mainly used in offline rendering. Nevertheless, some studies have shown significant optimization for methods which can render in real-time. Ramamoorthi et al. [7] presented an analytical expression for irradiance environment maps under the Spherical Harmonic (SH) coefficients of lighting. Debevec [5] used a technique introduced in his previous work [3] to acquire the correct calculation of scene incident light using multiple radiance maps through a variance cut algorithm. Then, he used this calculation to illuminate the synthetic objects with arbitrary material properties.

\subsection{Reflected Light and Global Illumination}

A photo-realistic rendering must address the indirect illumination between the objects which was contributed by the inter-reflections of light between surfaces in the final scene. Since Keller [4] introduced Instant Radiosity in 1997, it was developed to replace the Virtual Point Lights (VPLs) in order to approximate the reflected lights. The current hardware would be suitable for this method which does not require excessive pre-computations. Knecht et al. [10] combined the instant radiosity with differential rendering which renders the scene once for plausible realistic mixed reality systems while maintaining a frame rate of 30fps interactively. Kán et al. [?] estimated the indirect illumination from a photon map by performing a density estimation on every hit diffuse surface at the raytracing rendering. Gruber et al. [16] reconstructed a scene geometry using an RGB-D sensor that supports fast updating and static scene geometry that in turn enables user interaction with reflected lights. 


\subsection{Panoramic $360^{\circ}$ Live-feed}

The deployment of a $360^{\circ}$ panoramic video has appeared in Virtual and Mixed Reality (VR/MR) applications. In some game engines, a pre-recorded video was provided for immersive experience and realistic lighting in the dynamic environments. Rhee et al. [17] developed an immersive system for interactive mixed reality which used a Low Dynamic Range (LDR) $360^{\circ}$ video on Head-Mounted Displays (HMDs) where IBL was optimized to provide a fast and correct lighting source information. Fan et al. [18] studied the prediction of Field-of-Views (FoVs) when the viewer was watching $360^{\circ}$ video using HMDs. However, it is rare to find studies that deploy a $360^{\circ}$ panoramic video in augmented reality.

\section{Method and Implementation}

A quick overview of the system components are described in this section where a coherent final scene is produced in dynamic environments. For a more organized flow, the system components are examined in four subsections although they work simultaneously in real-time. A visualized version of the overview is shown in Figure 3.

Polarization of $360^{\circ}$ Live-feed. The white surfaces, unwanted reflections, and glare that was confusing our light detection algorithm in our previous work are reduced or absorbed completely after polarizing the incident light from the physical environments.

Detection of Incident Lights. The polarized lights reaching the $360^{\circ}$ camera view are investigated through computer vision sampling methods to calculate the angle and direction of multiple physical lights.

Estimation of Reflected Lights. The lights bouncing between the virtual and real objects are extracted from the local regions surrounding each object of concern and then applied through the image-based lighting mode.

Creation of Shading Properties. The virtual object materials and features are defined in this section where specific shaders are created in order to meet the requirements of reading from a live-feed cameras.

Rendering. Differential rendering is used and enhanced to perform with lower computational cost in real-time. The direct and indirect illumination are addressed separately for differential rendering shown in Figure 3. The rendering paths include normal maps and specular maps per-pixel lighting where any additional passes would not be necessary for reflection depth and normal buffers.

Tracking. The positional device tracker supported by Vuforia AR engine was used for a robust 6 degree-of-freedom (DOF) target tracking. Yet, an additional modifications were added through separate scripts to support the lighting conditions in our system when the objects, camera, and marker are changing locations.

Hardware Description. The main device used to test the system has Intel@ Core $^{\mathrm{TM}} \mathrm{i} 7-3930 \mathrm{k} \mathrm{CPU} @ 3.20 \mathrm{GHz} 3201 \mathrm{MHz}$, six core(s), 64.0 GB RAM, and NVIDIA GeForce GTX 970 GPU. For data input devices a DSLR Nikon D7200 was used as the main AR camera and a live-feed RICOH THETA S $360^{\circ}$ 
is dedicated to read the environment maps. Three Polarizing films (filters) were used to reduce reflections and glare.

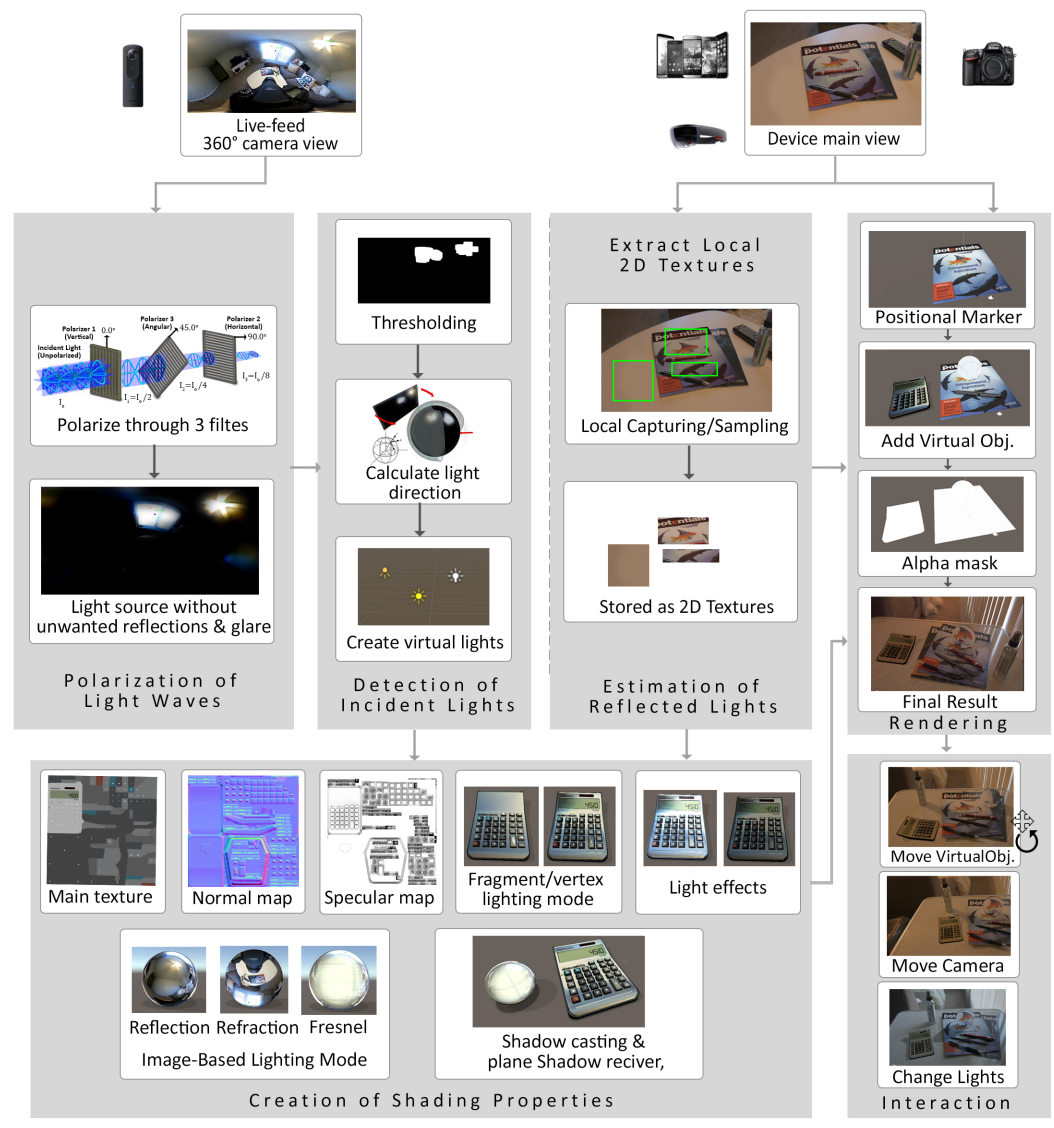

Fig. 3. An overview of the entire system components: Polarization of $360^{\circ}$ Live-feed, detection of incident light, simulation of reflected light, and creation shading property followed by rendering and interaction.

\subsection{Polarization of $360^{\circ}$ Live-feed}

Natural and artificial illumination yield light waves with electric field vectors that lie in planes oriented in every direction that is perpendicular to the propagation direction. The polarization of light is based on the quantum state of a photon described in $[9,13,2]$. In this section, the polarized filters are investigated in order to focus only on the genuine light sources while reducing unwanted reflections and glares which could be mistaken as light sources. Three polarizing film sheets 
are used as an initial component of the system. The light transmitted through the first filter is vertically polarized, while a second polarizer perpendicular to the first one is added to absorb the polarized light passing through the first film. However, the interesting part is that when a third filter is placed between the previous two perpendicular polarizers at a $45^{\circ}$ angle, it allows some of the light to get through the last filter (see illustration in Figure 4).

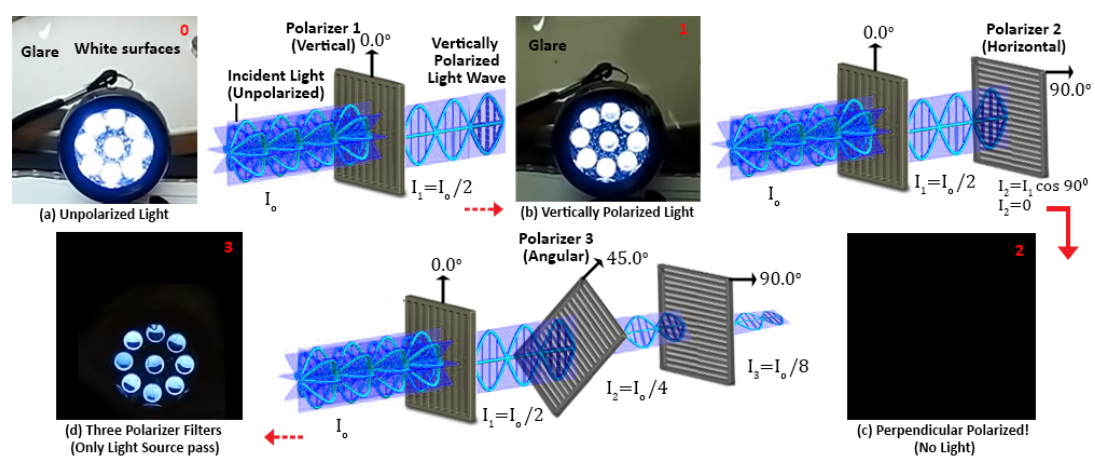

Fig. 4. Polarization of light waves through three filters: Vertical, Horizontal, and angular in order to capture the light source only.

The matrix representations can model the different types of polarizers as vectors. The vertical $|\uparrow\rangle\langle\uparrow|$, horizontal $|\rightarrow\rangle\langle\rightarrow|$, and angular $|\nearrow\rangle\langle\nearrow|$ directions can be represented as the following vectors:

$$
|v\rangle=\left(\begin{array}{l}
1 \\
0
\end{array}\right),|h\rangle=\left(\begin{array}{l}
0 \\
1
\end{array}\right),|\Theta\rangle=\left(\begin{array}{c}
\cos (\theta) \\
\sin (\theta)
\end{array}\right)
$$

Let the intensity of unpolarized light be noted as $I_{o}$. Then the polarized light passing though the three filters can be illustrated using Dirac notation for the photons. The vertically polarized light transmitted through the first film is $I_{1}=$ $\frac{I_{o}}{2},|\uparrow\rangle=\frac{1}{\sqrt{2}}[|\nwarrow\rangle+\langle\nearrow|]$. This is followed by the photons passing through the second polarizer: $I_{2}=\frac{I_{o}}{4},|\nearrow\rangle=\frac{1}{\sqrt{2}}[|\uparrow\rangle+\langle\rightarrow|]$, thus, producing the final notation as: $I_{3}=\frac{I_{o}}{8},|\rightarrow\rangle$.

The basic principle of matrix mechanics is used to examine the three polarizers' mathematical apparatus. The unpolarized light vibrating from the light source is a combination of every polarization angle from 0 to $\pi$ radians. The absolute magnitude square to the probability amplitude represents the final probability. Thus, the amount of light passing through the vertical polarizer is 0.5 which results from integrating every angle, and with $\frac{1}{\pi}$ as the normalization constant, the value can be calculated as:

$$
\frac{1}{\pi} \int_{0}^{\pi}\left|\left(\begin{array}{ll}
1 & 0
\end{array}\right)\left(\begin{array}{ll}
0 & 0 \\
0 & 1
\end{array}\right)\left(\begin{array}{c}
\cos (\theta) \\
\sin (\theta)
\end{array}\right)\right|^{2} d \theta=0.5
$$


As mentioned above, for the vertical polarized light, passing through the horizontal filter, both perpendicular to each other, the probability is shown as:

$$
\frac{1}{\pi} \int_{0}^{\pi}\left|\left(\begin{array}{ll}
0 & 1
\end{array}\right)\left(\begin{array}{ll}
0 & 0 \\
0 & 1
\end{array}\right)\left(\begin{array}{ll}
1 & 0 \\
0 & 0
\end{array}\right)\left(\begin{array}{l}
\cos (\theta) \\
\sin (\theta)
\end{array}\right)\right|^{2} d \theta=0
$$

Yet, when a third $45^{\circ}$ angled filter is sandwiched between the vertical and horizontal filters, the final probability shows the final unpolarized light

$$
\frac{1}{\pi} \int_{0}^{\pi}\left|\left(\begin{array}{ll}
0 & 1
\end{array}\right)\left(\begin{array}{ll}
0 & 0 \\
0 & 1
\end{array}\right)\left(\begin{array}{ll}
0.5 & 0.5 \\
0.5 & 0.5
\end{array}\right)\left(\begin{array}{ll}
1 & 0 \\
0 & 0
\end{array}\right)\left(\begin{array}{l}
\cos (\theta) \\
\sin (\theta)
\end{array}\right)\right|^{2} d \theta=0.125
$$

This probability represents the whole polarized physical scene where most of the information is lost but the incident lights in the real world can be detected in a practical approach as shown in the next section. This concept can be utilized in designing and making the necessary hardware to help in detecting direct illumination sources in scenes. The major advantage of using polarization is that it is a fast and practical method for the determination of the automatic statistical thresholds. This is in addition to producing better color fidelity of the real light source. On the other hand, polarizing filters are additional physical resources required for the system, and the environment maps after polarization filtering cannot be used for indirect illumination simulation.

\subsection{Detection of Incident Lights}

The live-feed from the panoramic $360^{\circ}$ camera produces a continuous radiance map that can be sampled efficiently. For a dynamic environment map that runs in real-time, the luminance pixels are captured using a thresholding approach. An automatic threshold value is given, and a pixel is considered as part of a light source if its intensity is above the threshold. A mask is produced that only depicts the area of light sources. The radiance map image is converted to gray-scale to ensure connectivity in white color-space. In order to determine the threshold automatically, two approaches are considered: (1) Calculate the median of the radiance map histogram because the temporal and spatial variations of the realtime environment change drastically which will affect the overall threshold value. (2) The polarized light allows only a small amount of incident light to pass through. Thus, the range of threshold is expanded significantly to a static binary value which performs faster in real-time.

In order to reduce the noise among the sampled points, Gaussian Blur is applied before thresholding. An erosion morphological transformation was applied with a rectangular kernel in small size to discard the pixels near boundaries of the mask. This is followed by a dilation transformation with a kernel triple in size to that used in the erosion. The pixel's luminance indicates the incident light in the radiance map which was extracted as a series of regions based on their area size to define the main light first, then the second light and so on. Identifying the color of the light provides more realistic illumination information which is 
computed based on the mean color for collective pixels in each region area. The resulting color is more accurate when the polarizing filters are used where the incident lights are the main objects. The error metric for the pixel of basic color $P b$ and the polarized one $P p$ is: $\sum_{\text {Pixels }}\left|\left(R_{b}, G_{b}, B_{b}\right)-\left(R_{p}, G_{p}, B_{p}\right)\right|$.

The intensity of the pixels also is calculated relative to the entire radiance map which is used to determine the soft and hard shadow strength based on the light type. The centroid of the sampled regions is converted from the screen coordinates $(x, y)$ to the spherical coordinates $(\theta, \phi)$ using the inverse spherical projection after normalization as shown in Figure 3 in the second step of detecting the incident light. The spherical coordinates are represented as: $(\theta, \phi)=\int_{1}^{\text {lights }}\left(\tan ^{-1} \frac{y}{x}, \cos ^{-1} \frac{z}{r}\right)$. Where $r=\sqrt{x^{2}+y^{2}}$ and lights is the number of lights detected in the panoramic $360^{\circ}$ view which influences the inverse spherical projection. Eventually, the virtual light that represent the location of the detected incident light is represented as follows:

$$
(x, y, z)=\int_{1}^{l i g h t s}\left(\frac{\sin \phi \cos \theta}{\pi \times d h}, \frac{\cos \phi}{\pi \times d w}, \frac{\sin \phi \cos \theta}{\pi \times 90}\right)
$$

However, these coordinates have a negative direction in the reverse direction of the view as shown:

$$
(x, y, z)=\int_{1}^{l i g h t s}\left(\frac{\sin \phi \cos \theta}{\pi \times(d h-90)},-\frac{\cos \phi}{\pi \times(d w-180)}, \frac{\sin \phi \cos \theta}{\pi \times 90}\right)
$$

These created virtual lights are normalized for the $3 \mathrm{D}$ engine where quaternions are used to represent the orientation or rotation of the lights. Also, when the light is turned off it can easily be deactivated.

\subsection{Estimation of Reflected Lights}

This section describes how the extraction of the reflected lights is performed. These type of light is also known as indirect illumination or reflected light which simply simulates any reflective interactions among the real and virtual object surfaces. The area surrounding the virtual objects from the $360^{\circ}$ live-feed view is captured to depict any lighting variations and instantly reflected on the virtual objects' shading properties in the IBL mode. The virtual object is attached with a $3 \mathrm{D}$ plane to capture the local area as a $2 \mathrm{D}$ texture. The mesh vertices of the $3 \mathrm{D}$ plane use a filter to keep updating the value at $100 \mathrm{fps}$ rate. In order for the object layer to only read the texture in the area of interest, a culling mask is used where the other parts of the main view are ignored. The mesh of the background plane dispatches every $10 \mathrm{fps}$ to obtain the main texture and assign it to the video texture. The resulting mesh has transformed vertices from the $3 \mathrm{D}$ world to the view port of each point. The orientation of the AR device is modified with the screen orientation. A renderer on a virtual camera captures the targeted texture and uploads it to IBL defined texture property. The quality of the local texture is normalized based on the current standards of the entire AR view. This simulation is perfect for diffuse and glass materials as shown in Figure 1. 


\subsection{Creation of Shading Properties}

Most current 3D engines provide predefined shaders through the CG language and OpenGl. However, due to the specific purposes of the current system that deals with a live-feed $360^{\circ}$ camera, multiple unique shaders are created to meet the system's needs. Each virtual object is a collection of vertices that consist of UV data, normals, and more. Some of the object material features can be precomputed such as: main texture, normal map, specular map, while other can be computed at run-time; even the predefined properties can be influenced by the real-time properties. There are two main type of shaders used in this system:

Surface Shader. The virtual objects that reflect the light and have material features are assigned to this shader. Three passes are used but not necessarily in every rendering frame: (i) fast forward base for the main incident light, (ii) forward add for any additional lights, and (iii) shadow caster. The created shader allows the vertex of fragment rendering options for the lighting mode. It provides local additional reflected lights and other properties such as Lambert Diffuse, Blinn Phong Specular, IBL Reflection, IBL Refraction, IBL Fresnel.

Unlit Shader. The virtual objects that cast shadow only without having any materialistic lights have this shader. For instance, the background on each placed virtual object must reflect shadows on the surfaces under them or they will look like floating objects in the air. Thus, to cut through the plane surfaces, an alpha mask is used to make the background planes have a transparent material but receive shadows from the other objects.

\section{Results and Evaluation}

Several environments, lighting conditions, and locations are tested through the evaluation process in order to ensure that our system, as promised, has a visually Coherent Augmented Reality scene. Some example of the results are presented in Figure 5.

\subsection{Direct Illumination Evaluation}

The shadow cast from the virtual objects is an indication of the incident light location when it is compared with the shadow cast from the real object. Therefore, the basic approach of calculating the direction of the sunlight is used in this step of the evaluation process. The real object's shadow $s$ and height $h$ provides the required inputs to calculate the angle $\theta$ that indicates the location of the light source as $\theta=\tan ^{-1} \frac{h}{S}$. The angle of incident light is calculated manually in the physical scene. The measured angle then listed aligns with the corresponding detected angle $\phi$ that is obtained through the methods explained in section 3.1 and 3.2. Table 1 shows the small amount of error as calculated in degrees between both angles. The error statistics is averaged using the root-mean-square-error (RMSE) given by the formula: $R M S E=\sqrt{\frac{\sum_{i=1}^{6} \text { Error }_{i}^{2}}{6}}=1.567$. This resulting average is slightly improved compared to the older version of our system which did not include polarization [20,21]. 


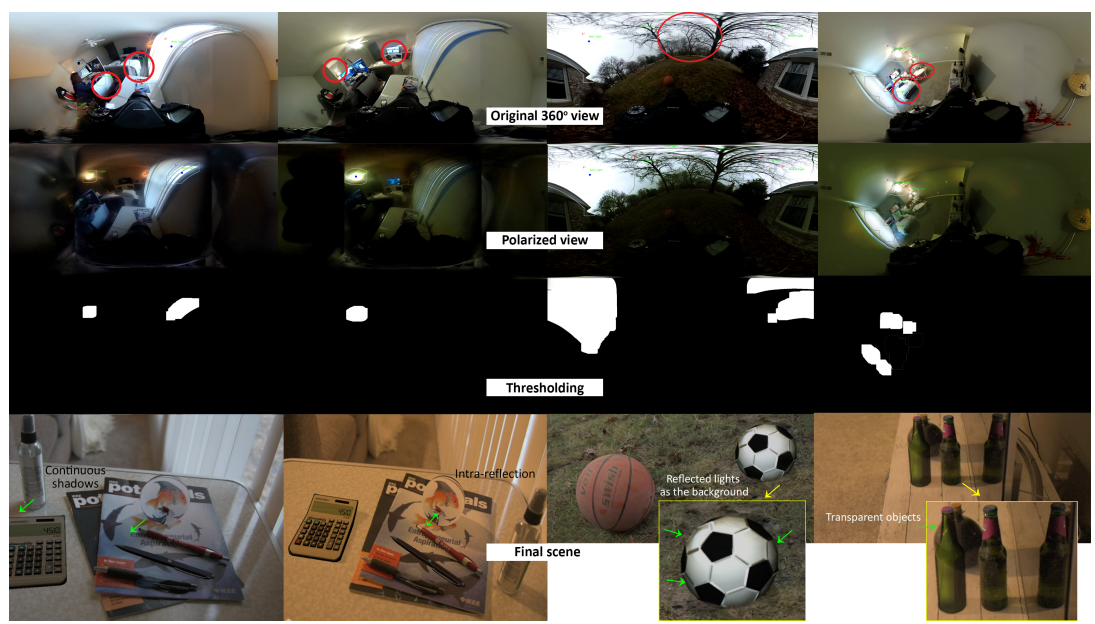

Fig. 5. System results with different lighting conditions and locations where each case has: (1) Original $360^{\circ}$ view "the red circles indicate the locations of unwanted reflections and glares mistaken with light sources", (2) the view after polarization, (3) Threshold with minimal requirements, (4) the final image of the scene.

Table 1. The measured angle $\theta$ compared with detected angle $\phi$ in degrees and the corresponding errors.

\begin{tabular}{|l||l|l|l|l|}
\hline Scene No. & Shadow length $\left(s^{\prime \prime}\right)$ & Measured $\left(\theta^{\circ}\right)$ & Detected $\left(\phi^{\circ}\right)$ & Error \\
\hline 1 & 26 & -169.13 & -168.05 & -1.08 \\
2 & 4.3 & 229.15 & 230.06 & -0.91 \\
3 & 14 & 19.61 & 18.55 & 1.06 \\
4 & 3 & -120.97 & -120.04 & -0.93 \\
5 & 16 & 17.32 & 15.2 & 2.12 \\
6 & 7 & 35.52 & 36.45 & -0.93 \\
\hline
\end{tabular}

\subsection{Performance Evaluation}

Global illumination has a major influence on the performance cost of a AR/MR system. Our previous work investigated several methods that can calculate the reflected lights with minimal cost. These methods include cube map (CM) [21], 2D Textures Sub-Sampling (2D) [20], and polarization (PZ) that is also compared with the methods presented in $[12,14,16]$ (GR12, GR14, and GR15, respectively; see Table 2). The categories of interest include: (FPS) number of frame per second where our system has achieved the required range of (30fps/60fps) for real-time execution, (Update) describes the process of the entire pipeline in [ms], (Input) is about the data captured through the main camera and the $360^{\circ}$ view. (Tracking) is involved with geometry reconstruction and 6-DOF camera tracking, the (Surfaces) involves the surfaces extracted by the renderer including occlusion computation. (Rendering) the time used for the virtual objects rasterization, differential rendering and the composition of AR scenes. 
Table 2. Performance evaluation compared to our previous methods: Cube Map (CM), 2D Textures Sub-Sampling(2D), with the current method polarization (PZ), also compared against methods from other research (GR12, GR14, GR15).

\begin{tabular}{|l||l|l|l||l|l|l|}
\hline \multirow{2}{*}{ Operation } & \multicolumn{3}{c||}{ Related Work } & \multicolumn{3}{c|}{ Ours } \\
\cline { 2 - 7 } & GR12 & GR14 & GR15 & CM & $2 \mathrm{D}$ & PZ \\
\hline FPS[1/s] & 5.8 & 12.29 & 22.46 & 6 & 45 & 50 \\
Update[ms] & 172.4 & 81.36 & 44.53 & 173.4 & 35.9 & 32.4 \\
Input[ms] & 7.3 & 7.44 & 7.02 & 0.04 & 0.05 & 0.06 \\
Tracking[ms] & 11.1 & 10.24 & 10.64 & 2.31 & 1.9 & 1.10 \\
Surfaces[ms] & 6.69 & 6.69 & 12.6 & 4.21 & 4.42 & 4.56 \\
Rendering[ms] & 0.92 & 0.98 & 0.87 & 0.51 & 0.43 & 0.32 \\
\hline
\end{tabular}

\section{Conclusion and Future Work}

In depth, the study of light and the physics of light are the keys aspect to rendering a realistic looking scene in computer graphics in general. In this work, we combined the idea of light wave polarization in physics and photography with a new platform represented in AR. The confusion of reflections and glare where they were mistaken with the light sources was eliminated after passing the light through the three polarizing filters (vertical, diagonal, and horizontal), which allowed the direct illumination to pass through and get detected instantly. The indirect illumination and object materials were addressed to ensure a visually coherent scene for the perception study. Although the augmented scenes do not operate without tracking, further investigation into the depth map and localization would benefit our future work of mimicking the reflected lights to result in more realistic rendering that uses the depth of every real object available in relation with the virtual objects. Furthermore, we would like to explore more shading and physics-based material effects which can improve the overall realism of the scene.

\section{References}

1. Horn BK.: Obtaining shape from shading information. The psychology of computer vision, 115-55 (1975).

2. Zerner MC.: Semiempirical molecular orbital methods. Reviews in computational chemistry, 313-65 (1991).

3. Debevec, P. E., Andmalik, J.: Recovering high dynamic range radiance maps from photographs. In SIGGRAPH'97, pp. 369-378 (1997).

4. Keller A.: Instant radiosity. In Proceedings of the 24th annual conference on Computer graphics and interactive techniques, ACM Press/Addison-Wesley Publishing Co., pp. 49-56 (1997).

5. Debevec P.: Rendering Synthetic Objects into Real Scenes: Bridging Traditional and Image-based Graphics with Global Illumination and High Dynamic Range Photography, Proceedings of the 25th Annual Conference on Computer Graphics and In- 
teractive Techniques, SIGGRAPH'98, 0-89791-999-8,189-198,10, ACM, New York, NY, USA. (1998).

6. Chen H, Wolff LB.: Polarization phase-based method for material classification in computer vision, International Journal of Computer Vision, 28(1):73-83 (1998)

7. Ramamoorthi R, Hanrahan P.: An efficient representation for irradiance environment maps. In Proceedings of the 28th annual conference on Computer graphics and interactive techniques, pp. 497-500 (2001).

8. Debevec P.: Image-based lighting. IEEE Computer Graphics and Applications. 22(2):26-34 (2002).

9. Brom JM, Rioux F.: Polarized Light and Quantum Mechanics: An Optical Analog of the Stern-Gerlach Experiment. The Chemical Educator, 7(4):200-4 (2002).

10. Knecht, M., Traxler, C., Mattausch, O., Purgathofer, W. and Wimmer, M.: Differential instant radiosity for mixed reality. In Mixed and Augmented Reality (ISMAR), 9th IEEE International Symposium, pp. 99-107 (2010).

11. Kán, P. and Kaufmann, H., November.: High-quality reflections, refractions, and caustics in augmented reality and their contribution to visual coherence. In Mixed and Augmented Reality (ISMAR), 2012 IEEE International Symposium on, pp. 99-108 (2012).

12. Gruber L, Richter-Trummer T, Schmalstieg D.: Real-time photometric registration from arbitrary geometry. In Mixed and Augmented Reality (ISMAR), IEEE International Symposium, pp. 119-128 (2012).

13. Jie BK.: Physics of Quantum Key Distribution, CS2107-Semester IV, 107 (20142015).

14. Gruber, L., Langlotz, T., Sen, P., Hoherer, T. and Schmalstieg, D.: Efficient and robust radiance transfer for probeless photorealistic augmented reality. In IEEE Virtual Reality (VR), pp. 15-20 (2014).

15. Ngo Thanh T., Nagahara H., Taniguchi RI.: Shape and light directions from shading and polarization. In Proceedings of the IEEE Conference on Computer Vision and Pattern Recognition, pp. 2310-2318 (2015).

16. Gruber, L., Ventura, J. and Schmalstieg, D.: Image-space illumination for augmented reality in dynamic environments. In Virtual Reality (VR), pp. 127-134 (2015).

17. Rhee T, Petikam L, Allen B, Chalmers A.: Mr360: Mixed reality rendering for 360 panoramic videos. IEEE Transactions on Visualization and Computer Graphics, (4):1379-88 (2017).

18. Fan CL, Lee J, Lo WC, Huang CY, Chen KT, Hsu CH.: Fixation prediction for 360 video streaming in head-mounted virtual reality. In Proceedings of the 27th Workshop on Network and Operating Systems Support for Digital Audio and Video, pp. 67-72 (2017).

19. Shen L., Zhao Y., Peng Q., Chan JC., Kong SG.: An iterative image dehazing method with polarization. IEEE Transactions on Multimedia. (2018).

20. Alhakamy, A., Tuceryan, M.: AR360: Dynamic Illumination for Augmented Reality with Real-Time Interaction. In 2019 IEEE 2nd International Conference on Information and Computer Technologies ICICT, pp. 170-175 (2019).

21. Alhakamy, A., Tuceryan, M.: CubeMap360: Interactive Global Illumination for Augmented Reality in Dynamic Environment. In IEEE SoutheastCon (2019) (Accepted and presented). 